\title{
Segment Distributions of End-Tethered Polymers in a Good Solvent
}

\author{
R. Lehner, J. Koota, G. Maret, and T. Gisler* \\ Fachbereich Physik, Universität Konstanz, 78457 Konstanz, Germany
}

(Received 8 July 2005; published 13 March 2006)

\begin{abstract}
We use confocal fluorescence microscopy to study the conformation of single DNA molecules endtethered to a solid substrate. The segment distribution $\rho(z)$ measured for chains with contour lengths $15.4 \mu \mathrm{m} \leq L \leq 59.4 \mu \mathrm{m}$ as a function of the distance from the substrate $z$ can be scaled onto a master curve depending only on the scaled distance $z / R_{\mathrm{g}}$, in quantitative agreement with theoretical predictions for end-tethered polymers in a good solvent. The scaling of the radius of gyration $R_{\mathrm{g}} \sim L^{0.57 \pm 0.05}$ shows the presence of excluded-volume interactions between the charged DNA segments. Independent measurements of $R_{\mathrm{g}}$ from end-segment distributions are in good agreement with values obtained from the segment distributions and provide evidence that the radius of gyration of end-tethered chains in a good solvent is identical to that of the free chain.
\end{abstract}

PACS numbers: $61.25 . \mathrm{Hq}, 82.35 . \mathrm{Gh}, 82.35 . \mathrm{Rs}, 83.80 . \mathrm{Rs}$

Polymers tethered to solid substrates have widespread technical applications, such as the stabilization of colloidal suspensions against coagulation, the protection of biosensors against unspecific binding, and for lubrication and adhesion [1-4]. The presence of an impenetrable substrate profoundly affects the conformation and segment distribution of a polymer chain with $N$ segments that is attached to it by one of its ends at a tether surface density $\sigma$. When the interaction between monomers and substrate is repulsive, scaling theory for isolated end-tethered polymers predicts a depletion zone near the surface characterized by a segment distribution increasing as $\rho(z) \sim z^{\zeta}$ with the distance $z$ from the substrate; here, $\zeta$ is related to the critical (Flory) exponent $\nu$ by $\zeta=(1-\nu) / \nu$ which in a good solvent $(\nu=0.588$ [5]) takes the value $\zeta=0.7$ [6]. At larger distances, the influence of the wall should become weaker, and the segment distribution can be expected to approach a maximum value $\rho\left(R_{\mathrm{g}}\right) \approx \sigma N / R_{\mathrm{g}}$ given by the average segment density within the polymer layer whose height is approximately given by the radius of gyration $R_{\mathrm{g}}$ of the free, unperturbed chain [6]. The slow increase of the segment distribution close to the substrate, together with its fast decay for distances $z \gg R_{\mathrm{g}}$ predicted by renormalization-group (RG) calculations [7] and computer simulations [8], suggests that the segment distribution of an end-tethered polymer in the low-density limit $\sigma R_{\mathrm{g}}^{2} \ll 1$ is strongly asymmetric, resembling the shape of a mushroom.

Although this "mushroom" conformation of an isolated end-tethered polymer represents the simplest situation of a broken symmetry for polymer statistics, detailed experimental tests of the theoretical predictions for $\rho(z)$ in the low-density limit are lacking. Neutron reflectivity measurements on end-adsorbed diblock copolymers confirmed the existence of a depletion layer close to the surface and the RG prediction for the second moment of the segment distribution $\left\langle z^{2}\right\rangle=2.16 R_{\mathrm{g}}^{2}[9,10]$. However, the determi- nation of the segment distribution $\rho(z)$ and radius of gyration $R_{\mathrm{g}}$ of polymer mushrooms using neutron reflectivity is difficult since this technique requires the averaging over a large number of polymer chains whose length distribution is usually not negligible for synthetic polymers.

In this Letter we present direct measurements of the segment distributions of end-tethered polymers with uniform chain length in a good solvent, using confocal fluorescence microscopy on single DNA molecules labeled with a fluorescent intercalation dye. Segment distributions measured over the range of contour lengths $15.4 \mu \mathrm{m} \leq$ $L \leq 59.4 \mu \mathrm{m}$ can be superimposed on a master curve by scaling the distance $z$ from the surface with the radius of gyration $R_{\mathrm{g}}$. The measured radius of gyration is found to scale as $R_{\mathrm{g}} \sim L^{0.57 \pm 0.05}$, providing direct evidence for excluded-volume behavior due to electrostatic repulsion between the DNA segments. Independent measurements of $R_{\mathrm{g}}$ from end-segment distributions are in good agreement with values obtained from the segment distributions and provide evidence that the radius of gyration of end-tethered chains in a good solvent is identical to that of the free chain.

Experiments were carried out with double-stranded DNA molecules attached with one end to glass substrates [11]. A confocal microscope equipped with a $100 \times$ objective with numerical aperture 1.45 , a Nipkow disk and a cooled CCD camera (Hamamatsu Orca II) was used to measure the YOYO-1 fluorescence at $\lambda=(525 \pm 25) \mathrm{nm}$ with an integration time of $0.8 \mathrm{~s}$ per frame. Slices through the 3-dimensional distributions of the fluorescence intensity were measured from a position $z_{\min } \approx-2 \mu \mathrm{m}$ well below the substrate-solvent interface to $z_{\max } \approx 10 \mu \mathrm{m}$ above it, in intervals $\Delta z=100 \mathrm{~nm}$. Within the field of view of the confocal microscope of $35 \mu \mathrm{m} \times 45 \mu \mathrm{m}$ we typically observed about 10 DNA molecules simultaneously, corresponding to a very low reduced tether density $\sigma R_{\mathrm{g}}^{2} \approx 4 \times 10^{-4}$. All the surface-bound DNA molecules 
were end-attached, as shown by fluorescence images recorded during electrophoretic stretching of the DNA parallel to the substrate.

The fluorescence signal from the DNA molecules was isolated from the background signal and detector noise by defining areas of about $3 \mu \mathrm{m} \times 3 \mu \mathrm{m}$ around bright, fluctuating intensity maxima that could be identified as DNA molecules. High-frequency noise was removed by convolving each frame with a Gaussian 1.5 pixels wide. By selecting an area of $1 \mu \mathrm{m} \times 1 \mu \mathrm{m}$ in a DNA-free spot next to a molecule we estimated the local background signal for each molecule separately, which was then subtracted from the denoised data, yielding the denoised and background-corrected 3-dimensional fluorescence signal $R(\boldsymbol{r})$ [see Fig. 1(a)]. In order to measure the intensityweighted segment distribution $S(z)$ each of the $R(\boldsymbol{r})$ frames was binarized in the $x y$ plane. The threshold was defined by the mean intensity plus the standard deviation of the intensity distribution of the frame. The area $A(z)$ occupied by one molecule at height $z$ was determined from the bright pixels of the resulting black-and-white image. The integrated fluorescence intensity within this area was calculated and normalized with $A(z)$ resulting in the intensityweighted segment distribution $S(z)$. The origin of the $z$ axis was determined by locating the maximum in the background signal near the solvent-substrate interface arising from streptavidin-bound oligonucleotide traces. As shown in Fig. 1(b), the intensity-weighted segment distribution $S(z)$ from an end-tethered $\lambda$ DNA molecule exhibits a strongly asymmetric shape, quite unlike the symmetric profiles of colloidal spheres. At small distances $z$ from the surface, the fluorescence intensity increases from very small values to a pronounced maximum located at $z \approx 0.8 \mu \mathrm{m}$. At larger distances, the segment distribution shows a broad, markedly non-Gaussian tail. Indeed, small, albeit significant fluorescence signal from the polymer can still be detected at distances of $4 \mu \mathrm{m}$ which corresponds to about $5 R_{\mathrm{g}}$ for this chain length.
RG theory and simulations predict that the segment distribution of an isolated end-tethered polymer in a good solvent follows a scaling form $\tilde{\rho}\left(z / R_{\mathrm{g}}\right)$, the scaling factor being the radius of gyration $R_{\mathrm{g}}$ of the free (untethered) chain $[7,8]$. In order to test this prediction, we measured the intensity-weighted segment distribution $S(z)$ for endtethered DNA with contour lengths $8.2 \mu \mathrm{m} \leq L \leq$ $59.4 \mu \mathrm{m}$, corresponding to chains with $20040 \leq N_{0} \leq$ 145506 basepairs [12].

Lacking a closed-form expression for $\rho(z)$ for general values of the excluded-volume parameter [7], we determine the radius of gyration $R_{\mathrm{g}}$ using the expression

$$
\rho(z)=\frac{1.786 \sigma N}{R_{\mathrm{g}}}\left(\frac{z}{R_{\mathrm{g}}}\right)^{-0.3}\left[\operatorname{erfc}\left(\frac{z}{2 R_{\mathrm{g}}}\right)-\operatorname{erfc}\left(\frac{z}{R_{\mathrm{g}}}\right)\right]
$$

proposed by Kreer et al. [8] to interpolate between the behavior of end-tethered excluded-volume chains at small and at large distances $z . \rho(z)$ is related to the propagator of the $n$th segment of the tethered chain, $G_{n}(\mathbf{0}, \boldsymbol{r})$ [14], by $\rho(z)=\sigma \int d x d y \int_{0}^{N} d n G_{n}(\mathbf{0}, \boldsymbol{r})$. Equation (1) was shown to be in excellent agreement with RG theory and simulations and allows us to cast all excluded-volume effects into the radius of gyration $R_{\mathrm{g}}$ of the free chain. We measure $R_{\mathrm{g}}$ by fitting the convolution product $B \int_{z_{\min }}^{z_{\max }} \rho\left(z-z^{\prime} ; R_{\mathrm{g}}\right) \times$ $K_{z}\left(z^{\prime}\right) d z^{\prime}$ to the measured $S(z)$ [13], where $B$ is a free parameter and $K_{z}(z)$ is the measured longitudinal pointspread function [15].

Figure 2 shows that when the intensity-weighted segment distributions $S(z)$ (normalized to unit area) are plotted vs the rescaled distance $z / R_{\mathrm{g}}$, the data for the different contour lengths superimpose onto a master curve. While the data of the shortest chains $\left(N_{0}=20040, L=8.2 \mu \mathrm{m}\right)$ show some deviations from scaling, possibly due to the limited resolution of the microscope, the scaling of the distributions of the longer chains provide quantitative evidence that the profiles are governed by a common chain statistics.

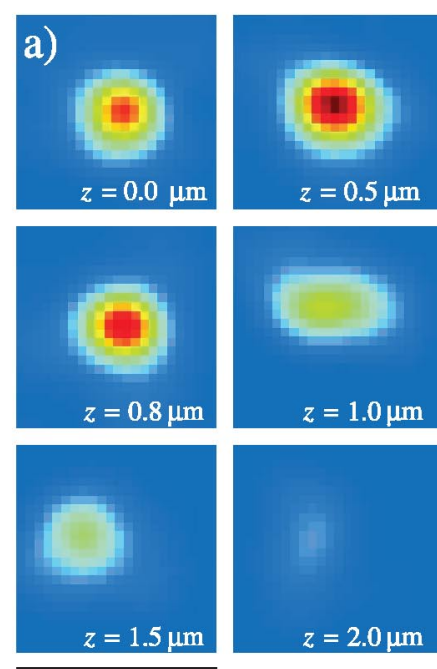

$3.0 \mu \mathrm{m}$

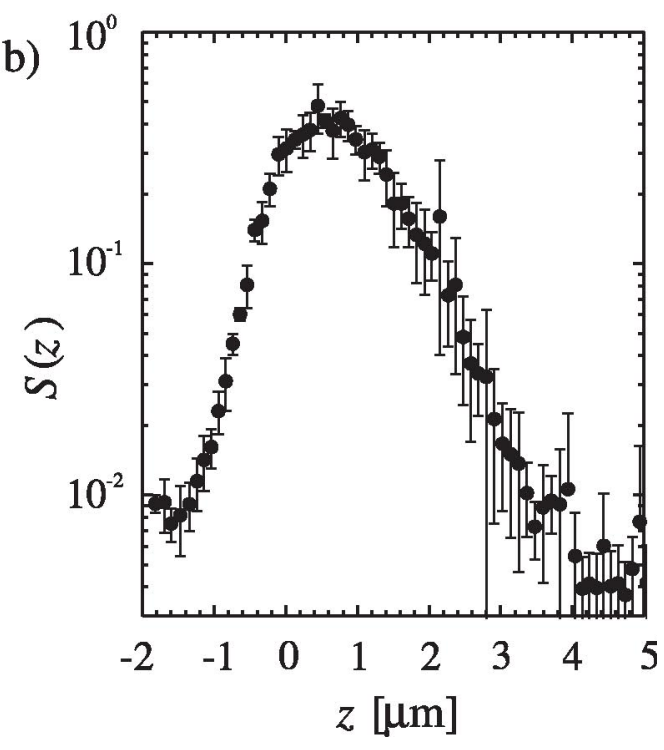

107801-2
FIG. 1 (color). (a) Cuts through the denoised and background-corrected fluorescence signal $R(\boldsymbol{r})$ of an end-tethered $\lambda$ DNA molecule at different heights $z$ from the substrate. (b) Intensityweighted segment distribution $S(z)$ of isolated end-tethered $\lambda$ DNA with contour length $L=19.8 \mu \mathrm{m}$, as a function of the distance $z$ from the substrate. Error bars represent the standard deviation of the measurements over 10 molecules. A common $z$ axis was obtained by binning the $z$ axes from the 10 data sets, using a binning width of $0.1 \mu \mathrm{m}$. The extension of the profile to negative values of $z$ is due to the finite resolution of the microscope. 


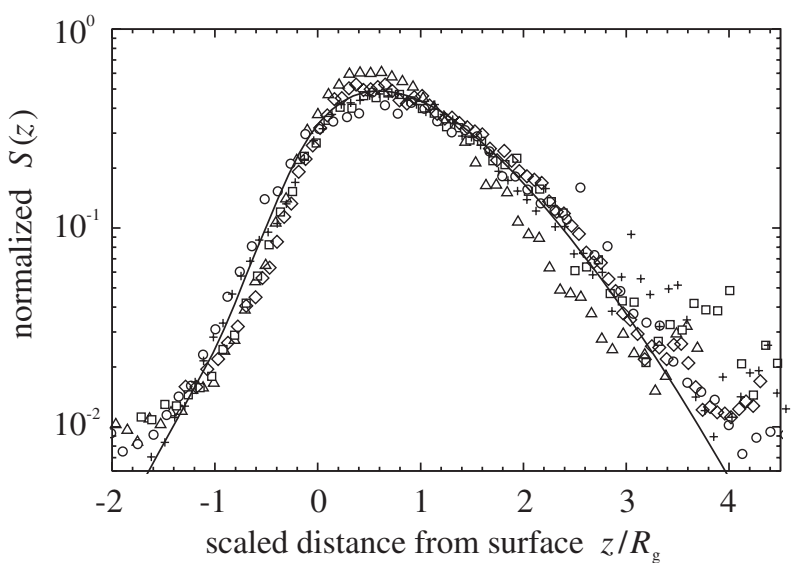

FIG. 2. Intensity-weighted segment distribution $S(z)$, normalized by $\int_{z_{\min }}^{z_{\max }} S(z) d z$, of end-tethered DNA as a function of the reduced distance $z / R_{\mathrm{g}}$ from the substrate. Symbols: data for DNA with $N_{0}=20040$ (triangles), $N_{0}=38416$ (squares), $N_{0}=48502$ (circles), $N_{0}=97004$ (crosses), and $N_{0}=$ 145507 (diamonds). Scaling factors $R_{\mathrm{g}}$ are determined from a fit of Eq. (1) to the data (see text). The full line is the scaling prediction Eq. (1) for $\tilde{\rho}\left(z / R_{\mathrm{g}}\right)$, convolved with the experimental resolution function $K_{z}(z)$.

In order to test whether the values of $R_{\mathrm{g}}$ determined from the segment distributions are consistent with those expected for free excluded-volume chains, we measure $R_{\mathrm{g}}$ for the contour lengths $15.4 \mu \mathrm{m} \leq L \leq 59.4 \mu \mathrm{m}$ which show good scaling with the excluded-volume theory Eq. (1) [16]. Figure 3 shows a power law scaling $R_{\mathrm{g}} \sim$ $L^{\nu}$ with an exponent $\nu=0.57 \pm 0.05$ which is in very good agreement with the critical exponent $\nu=0.588$ for

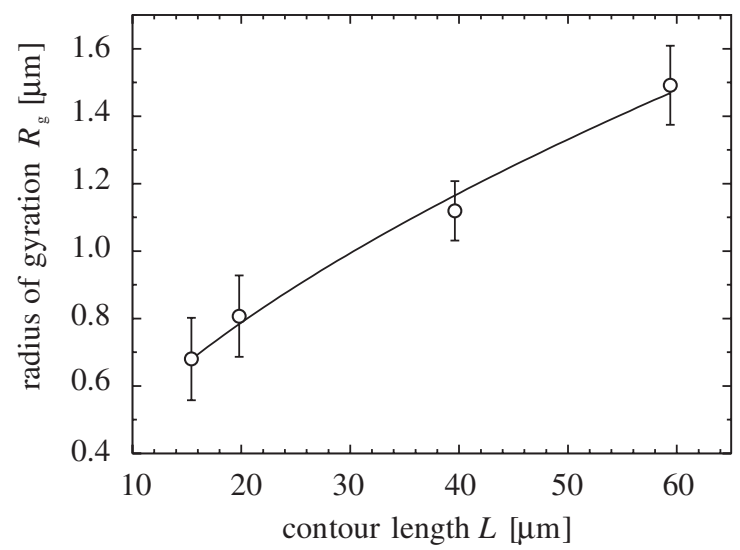

FIG. 3. Radius of gyration $R_{\mathrm{g}}$ determined from the measured intensity-weighted segment distributions $S(z)$, as a function of the contour length $L$ of DNA labeled with YOYO-1. Error bars represent the standard deviation of the measurements over 10 molecules. The line is a nonlinear least-squares fit of the power law $R_{\mathrm{g}}=B L^{\nu}$ to the data, with the best-fit exponent $\nu=0.57 \pm$ 0.05 . The contour length $L$ was calculated from the number of basepairs, $N_{0}$, and the basepair rise $a=3.4 \AA$ by the relation $L=1.2 N_{0} a$, the prefactor 1.2 taking into account the increase of the basepair rise upon YOYO-1 binding [16]. excluded-volume interactions. This provides clear evidence that the excluded-volume interactions are indeed governing the structure of isolated DNA chains in water at a $p \mathrm{H}$ and salinity compatible with biological function.

Similar to the segment distribution function $\rho(z)$, the end-segment distribution function $\rho_{\mathrm{e}}(z)=$ $\sigma \int d x d y G_{N}(\mathbf{0}, \boldsymbol{r})$ of end-tethered chains in a good solvent is also expected to follow a scaling behavior. Closed-form expressions for $\rho_{\mathrm{e}}(z)$ based on RG calculations or simulations are not known, but the similarity of the values of $R_{\mathrm{g}}$ obtained by analyzing our data with and without taking account of excluded-volume leads us to expect that the expression

$$
\rho_{\mathrm{e}}(z) \propto \exp \left[-\left(\frac{z-z_{0}}{2 R_{\mathrm{g}, \mathrm{e}}}\right)^{2}\right]-\exp \left[-\left(\frac{z+z_{0}}{2 R_{\mathrm{g}, \mathrm{e}}}\right)^{2}\right]
$$

for the end-segment distribution of an ideal, end-tethered chain [20] should yield values for the scaling factors $R_{\mathrm{g}, \mathrm{e}}$ that are similar to those expected for expanded chains.

To answer the question whether the scaling factor $R_{\mathrm{g}, \mathrm{e}}$ is identical with the radius of gyration $R_{\mathrm{g}}$ obtained from $\rho(z)$, we measured the intensity-weighted end-segment distribution $S_{\mathrm{e}}(z)$, using end-tethered $\lambda$ DNA to whose second, free end an avidin-coated fluorescent bead with diameter $100 \mathrm{~nm}$ (TransFluoSpheres, Molecular Probes) had been attached [13] (see Fig. 4). In order to distinguish the signal from the bead from that of the inner chain segments, the fluorescence of the bead is measured at $\lambda=605 \mathrm{~nm}$ where the fluorescence of DNA-bound YOYO-1 is very weak.

We use the expression for the ideal chain, Eq. (2), to determine the radius of gyration $R_{\mathrm{g}, \mathrm{e}}$ from the measured end-segment profiles $S_{\mathrm{e}}(z)$, approximating $z_{0}$, the position of the first monomer, by the persistence length $p$. Choosing $z_{0}=53 \mathrm{~nm}$ [17] results in a value $R_{\mathrm{g}, \mathrm{e}}=0.77 \mu \mathrm{m}$ for $\lambda$ DNA with 0.2 YOYO-1/bp dye loading which is in good agreement with the value $R_{\mathrm{g}}=0.8 \pm 0.1 \mu \mathrm{m}$ obtained from the analysis of $S(z)$. In the range of chain lengths studied here, the exact value of $z_{0}$ does not affect the
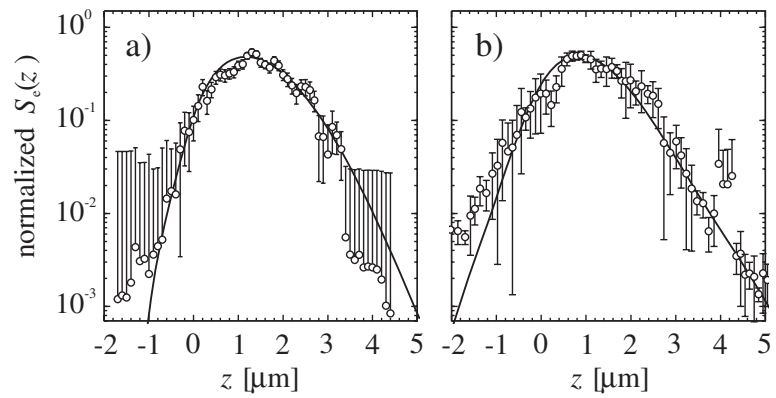

FIG. 4. Intensity-weighted end-segment distribution $S_{\mathrm{e}}(z)$ of a $\lambda$ DNA mushroom, as a function of the distance $z$ from the substrate. Error bars represent the standard deviation over 3 molecules. (a) DNA labeled with 0.2 YOYO-1/bp. (b) Unlabeled DNA. Lines: Eq. (2) convolved with the experimental resolution function $K_{z}(z)$ for (a) YOYO-1-labeled DNA $\left(R_{\mathrm{g}}=\right.$ $0.77 \mu \mathrm{m})$ and (b) unlabeled DNA $\left(R_{\mathrm{g}}=0.66 \mu \mathrm{m}\right)$. 
numerical values of $R_{\mathrm{g}, \mathrm{e}}$. In contrast, the analysis of $S_{\mathrm{e}}(z)$ from unlabeled $\lambda$ DNA yields a reduced value $R_{\mathrm{g}, \mathrm{e}}=$ $0.66 \mu \mathrm{m}$. This value is in excellent agreement with the radius of gyration $R_{\mathrm{g}, \mathrm{f}}=0.7 \mu \mathrm{m}$ obtained from static light scattering on free $\lambda$ DNA [21]. While independent measurements of $R_{\mathrm{g}}$ of YOYO-1-labeled DNA are not available, our results provide strong indications that the radius of gyration of tethered excluded-volume chains is identical with that of free chains.

The increase in $R_{\mathrm{g}}$ by $17 \%$ induced by YOYO- 1 binding should, according to the scaling expression for the radius of gyration of free excluded-volume chains [22]

$$
R_{\mathrm{g}, \mathrm{f}}=L^{3 / 5} a^{1 / 5} p^{1 / 5} \tau^{1 / 5},
$$

originate mainly in the increase of the contour length due to binding, and much less to changes in the persistence length $p$, basepair step $a$, and solvent quality factor $\tau$. However, the increase in the contour length by $20 \%$ alone would increase $R_{\mathrm{g}}$ by only $11 \%$. Using a value for the persistence length $p=77 \mathrm{~nm}$ for YOYO-1-labeled DNA [23] (assuming an increase of $p$ linear with dye loading) and an increase of the average basepair rise $a$ to $4.1 \AA$, Eq. (3) predicts a reduction of the solvent quality factor $\tau$ by approximately $30 \%$ upon YOYO-1 binding. This reduction of $\tau$ might originate in the reduction of DNA surface charge upon binding of YOYO-1, resulting in an effective increase of the theta temperature.

In summary, the equilibrium segment distributions measured in this work provide the first direct experimental test of theoretical predictions for the conformation of endtethered polymers in a good solvent. The combination of confocal fluorescence microscopy with its ability to provide 3-dimensional, high-resolution images, and longchain, monodisperse DNA molecules that can be labeled selectively could open up new avenues for the study of adhesion and friction phenomena in polymer solutions, such as wall-slip and interfacial rheology.

We thank S. Siegel and I. Seuffert for help with the sample preparation, F. O. Fackelmayer and T. Meergans for preparative advice, and J. Baschnagel, C. Marques, and R. Tuinier for stimulating discussions. This work was funded by the Deutsche Forschungsgemeinschaft.

*Electronic address: thomas.gisler@uni-konstanz.de

[1] L. Léger, E. Raphaël, and H. Hervet, Adv. Polym. Sci. 138, 185 (2003).

[2] U. Raviv, S. Giasson, N. Kampf, J.-F. Gohy, R. Jérôme, and J. Klein, Nature (London) 425, 163 (2003).

[3] C. Jeppesen, J. Y. Wong, T. L. Kuhl, J. N. Israelachvili, N. Mullah, S. Zalipsky, and C. M. Marques, Science 293, 465 (2001).

[4] A. G. Moreira, C. Jeppesen, F. Tanaka, and C. M. Marques, Europhys. Lett. 62, 876 (2003).
[5] J. C. LeGuillou and J. Zinn-Justin, Phys. Rev. Lett. 39, 95 (1977).

[6] P. G. de Gennes, Macromolecules 13, 1069 (1980).

[7] M. Adamuti-Trache, W. E. McMullen, and J.F. Douglas, J. Chem. Phys. 105, 4798 (1996).

[8] T. Kreer, S. Metzger, M. Müller, K. Binder, and J. Baschnagel, J. Chem. Phys. 120, 4012 (2004).

[9] M.S. Kent, L.-T. Lee, B. Farnoux, and F. Rondelez, Macromolecules 25, 6240 (1992).

[10] J. B. Field, C. Toprakcioglu, L. Dai, G. Hadziioannou, G. Smith, and W. Hamilton, J. Phys. II (France) 2, 2221 (1992).

[11] Cover slips were coated with aminopropyl-triethoxysilane, glutardialdehyde, and streptavidin. $\lambda$ DNA $\left[N_{0}=48502\right.$ basepairs (bp)] was end-functionalized with biotin-labeled oligomers, separated from unreacted nucleotides using a Nick column into Tris-borate-EDTA buffer at $p \mathrm{H} 8.6$ containing an antibleaching agent $(0.7 \mathrm{mg} / \mathrm{m} \ell$ glucose oxidase, $0.04 \mathrm{mg} / \mathrm{m} \ell$ catalase, $5 \mathrm{mg} / \mathrm{m} \ell$ glucose, and $0.1 \mathrm{M}$ dithiothreitol). DNA was incubated with YOYO-1 for at least $60 \mathrm{~min}$. at room temperature. The concentrations were adjusted to a label density of $0.2 / \mathrm{bp}$. Drops of a DNA solution at a concentration of typically $0.5 \mathrm{ng} / \mu \ell$ were then placed onto the cover slips. Unbound DNA was removed by careful rinsing.

[12] Fragments with $N_{0}=20040$ and $N_{0}=38416$ were obtained by digestion of $\lambda$ DNA with NaeI and ApaI, respectively. Dimers and trimers of $\lambda$ DNA with $N_{0}=$ 97004 and $N_{0}=145506$ were obtained by annealing of linearized $\lambda$ DNA using T4 ligase [13].

[13] R. Lehner, Ph.D. thesis, Universität Konstanz 2005. http:// www.ub.uni-konstanz.de/kops/volltexte/2005/1505/.

[14] M. Doi and S.F. Edwards, The Theory of Polymer Dynamics (Oxford University Press, Oxford, 1988), 1st ed.

[15] We determined the point-spread function $K(\boldsymbol{r})$ by measuring the fluorescence intensity distribution of small fluorescent spheres with diameter $d=100 \mathrm{~nm}$ attached to the substrate. The resulting longitudinal point-spread function $K_{z}(z)=\int K(\boldsymbol{r}) d x d y$ was described by a Gaussian with a width $2 \sigma_{z}=0.651 \mu \mathrm{m}$.

[16] We measured the average end-to-end distance $R_{\text {ee }}$ of endgrafted $\lambda$ DNA labeled with YOYO-1 by electrophoretic stretching as a function of the electric field $E$ parallel to the surface of the substrate. Analyzing the $R_{\mathrm{ee}}(E)$ curves with the wormlike-chain model $[17,18]$ yields the best-fit value for the contour length $L=19.8 \mu \mathrm{m}$. This expansion by $20 \%$ over the value $L=16.5 \mu \mathrm{m}$ of unlabeled $\lambda$ DNA is consistent with the increase of the basepair separation of about $4 \AA$ upon YOYO-1 intercalation [19].

[17] J.F. Marko and E. D. Siggia, Macromolecules 28, 8759 (1995).

[18] S. Ferree and H. W. Blanch, Biophys. J. 85, 2539 (2003).

[19] F. Johansen and J. P. Jacobsen, J. Biomol. Struct. Dyn. 16, 205 (1998).

[20] Y. Lépine and A. Caillé, Can. J. Phys. 56, 403 (1978).

[21] J.R. Dawson and J.A. Harpst, Biopolymers 10, 2499 (1971).

[22] D. W. Schaefer, J.-F. Joanny, and P. Pincus, Macromolecules 13, 1280 (1980).

[23] S. R. Quake, H. Babcock, and S. Chu, Nature (London) 388, 151 (1997). 